\title{
Broken time-reversal symmetry in a Josephson junction with an Anderson impurity between a two-band and a single-band superconductor
}

\author{
Y. Avishai ${ }^{1,2, *}$ and T. K. $\mathrm{Ng}^{1, \dagger}$ \\ ${ }^{1}$ Department of Physics, Hong Kong University of Science and Technology, Clear Water Bay, Kowloon, Hong Kong \\ ${ }^{2}$ Department of Physics and Ilse Katz. Institute for Nanotechnology, Ben Gurion University, Beer Sheva, Israel
}

(Received 28 January 2010; published 2 March 2010)

\begin{abstract}
A Josephson junction consisting of an Anderson impurity weakly coupled on one side with a two-band superconductor and on the other side with a a single-band superconductor exposes a time-reversal breaking ground state within a finite domain of the $U-J$ plane (here $U$ is the correlation strength and $J$ is the coupling between the two bands). In this regime, the impurity magnetic moment is attenuated and order-parameter phase differences across the junction are neither 0 nor $\pi$. This indicates a fundamental and distinct role of strong correlations: driving a system into a time-reversal breaking ground state.
\end{abstract}

DOI: 10.1103/PhysRevB.81.104501

PACS number(s): 74.81.Fa, 73.40.Gk, 74.45.+c, 74.50.+r

\section{INTRODUCTION AND MOTIVATION}

Recently, there is a renewed interest in the physics of multiband superconductors which are believed to encode the physics behind the medium $T_{c}=39 \mathrm{~K}$ superconductor $\mathrm{MgB}_{2}$ (Refs. 1-3) and the new iron-based superconductors. ${ }^{4-6}$ Historically, interest in two-band superconductors is related to experiments suggesting that in some transition metals, there is a second mechanism responsible for pairing beyond that of electron-phonon interaction. ${ }^{7-10}$ For example, in $\mathrm{Nb}_{3} \mathrm{Sn}$ the isotopic effect is found to be very weak. ${ }^{11}$ Besides, it is suggested that interband coupling provides a possible mechanism for augmenting $T_{c \cdot} \cdot^{10,12}$

As was recently noticed, a useful setup for studying the physics of two-band superconductors is a Josephson junction involving two coupled superconductors $(1,2)$ on one of its sides and a single-band superconductor (3) on its other side. ${ }^{13}$ Within a Ginzburg-Landau formalism the order parameters $\psi_{1}, \psi_{2}$ are coupled among themselves by a term $-J \operatorname{Re}\left[\psi_{1} \psi_{2}^{*}\right]$ and to the third superconductor by tunneling $\Gamma_{n} \operatorname{Re}\left[\psi_{n} \psi_{3}^{*}\right](n=1,2)$. Quite unexpectedly, a time-reversal violating ground state (TRVGS) was found for $\Gamma_{1} \approx \Gamma_{2}$ due to frustration between the three order parameters, while otherwise, a time-reversal conserving ground state (TRCGS) prevails.

We are motivated by the question how would strong correlations affect the physics of such Josepshon junction, when the two order parameters in the two-band superconductor are out of phase (that is, an $s_{ \pm}$superconductor). Will new physics emerge when strong correlation is coupled with this additional phase frustration? Indeed, interesting physics induced by strong correlation emerges already in a Josephson junction with an Anderson impurity (of strength $U$ ) between single-band superconductors. ${ }^{14-17}$ Within a mean-field (Hartree-Fock) approximation ${ }^{15}$ which is reliable in the weak to intermediate $U$ regime when the Kondo temperature is small compared with the superconducting gap, ${ }^{15,16}$ it was shown that the effective Josephson coupling changes sign when $U$ exceeds a critical value $U_{c}$. The two superconductors are in phase $(\delta=0)$ at $U<U_{c}$, and are out of phase $(\delta=\pi)$ at $U>U_{c}{ }^{18}{ }^{18}$ This $0 \rightarrow \pi$ Josephson junction transition is associated with the formation of a large impurity magnetic moment for $U>U_{c}$.
The rest of the paper includes a description of the model and the main results (Sec. II), Hamiltonian and choice of parameters (Sec. III), analysis of results in Sec. IV followed by a brief discussion in Sec. V.

\section{MODEL AND MAIN RESULTS}

The system studied here is an Anderson impurity (level energy $\varepsilon$ and correlation strength $U$ ) weakly coupled on the left with a two-band superconductor (order parameters $\psi_{n}=\Delta_{n} e^{i \theta_{n}}, n=1,2$, and interband Josepshon coupling $\left.J\right)$ and on the right to a single-band superconductor (order parameter $\psi_{3}=\Delta_{3} e^{i \theta_{3}}$. This is briefly denoted as $2 B-U-1 B$ Josephson junction (see Fig. 1). We focus on the free energy, the nature of the ground state, the impurity magnetization and Josephson current. A TVRGS occurs if the free energy $\mathcal{F}\left[\boldsymbol{\theta} \equiv\left(\theta_{1}, \theta_{2}, \theta_{3}\right)\right]$ has a minimum at some point $\overline{\boldsymbol{\theta}}$ for which $\sin \left(\bar{\theta}_{n}-\bar{\theta}_{3}\right) \neq 0,(n=1,2)$ even in the absence of magnetic field (see Ref. 13 for the case of $2 B-1 B$ Josephson junction without Anderson impurity). On the technical part, the meanfield (Hartree-Fock) approach to the $1 B-U-1 B$ problem ${ }^{15,17}$ can be used to treat the $2 B-U-1 B$ system when $U$ is not too large. It enables the elucidation of the ground-state configuration $\overline{\boldsymbol{\theta}}(J, U)$ together with the free energy $\mathcal{F}(\overline{\boldsymbol{\theta}})$, the Josephson current $\mathcal{J}(\overline{\boldsymbol{\theta}})$ the impurity occupation $n(\overline{\boldsymbol{\theta}})$ and magnetization $m(\overline{\boldsymbol{\theta}})$. Note that there exist now two independent phase variables compared with just one in the case of $1 B-U-1 B$ junction. It implies a richer phase diagram for the $2 B-U-1 B$ system. Thus, when $\left|\bar{\theta}_{1,2}-\bar{\theta}_{3}\right|=0$ or $\pi$ we have

$$
\Delta_{1} \mathrm{e}^{\mathrm{i} \theta_{1} \underset{\mathrm{J}}{\underset{5}{s}} \Delta_{2} \mathrm{e}^{\mathrm{i} \theta_{2}}} \quad \Delta_{3} \mathrm{e}^{\mathrm{i} \theta_{3}}
$$

FIG. 1. Geometry of the $2 B-U-1 B$ junction: the two-band superconductor on the left is described by two order parameters $\Delta_{1} e^{i \theta_{1}}$ and $\Delta_{2} e^{i \theta_{2}}$ and an interband pairing potential $J$. The single-band superconductor on the right is encoded by a single-order parameter $\Delta_{3} e^{i \theta_{3}}$. Electrons can cross the junction only through hopping into and out of the Anderson impurity $U$ located between the superconductors. 
$(0,0)$ or $(0, \pi)$ junctions with a TRCGS while otherwise there is a TRVGS.

Our main results demonstrate that strong correlations affect the time-reversal symmetry of the ground state. More precisely, when the couplings $\Gamma_{1}, \Gamma_{2}$ between superconductors $(1,2)$ and the impurity are distinct (here we took $\left.\Gamma_{1} / \Gamma_{2}=2.5\right)$, two phases are identified in the $U-J$ plane, one for which there is TRCGS and one for which there is TRVGS. They are separated by a sharp border line. The TRVGS occurs for $U_{1}(J)<U<U_{2}(J)$ and for $0<J_{1}(U)<J<J_{2}(U)$. Here $U_{1}<U_{0}<U_{2}$ where $U_{0}=-2 \varepsilon$ is the particle-hole symmetric point and $J_{1,2}(U, \delta)$ are some critical thresholds. The physical content of the TRVGS phase (which is almost insensitive to the details of the other parameters) is remarkable, displaying a dip in the impurity magnetization and order parameter phase differences $\left|\bar{\theta}_{i}-\bar{\theta}_{j}\right| \neq 0, \pi$. This system is hence neither 0 nor $\pi$ junction.

\section{FORMALISM}

The mean-field Hamiltonian is written as, $H=H_{L}+H_{R}+H_{\mathrm{I}}+H_{\mathrm{imp}}$. The first two terms $H_{L}, H_{R}$ describe (within the BCS formalism) the two-band superconductor on the left $\left(H_{L}\right)$ and the single-band superconductor on the right $\left(H_{R}\right)$. Explicitly, in terms of quasiparticle (Nambu) field operators $\Psi_{n}^{\dagger}(\mathbf{r})=\left[\psi_{n \uparrow}^{\dagger}(\mathbf{r}), \psi_{n \downarrow}(\mathbf{r})\right]$ we may write $H_{L}=\sum_{n=1}^{2} H_{n}$ and $H_{R}=H_{3}$ with

$$
\begin{gathered}
H_{n}=\int \Psi_{n}^{\dagger}(\mathbf{r}) \mathcal{H}_{n} \Psi_{n}(\mathbf{r}) d \mathbf{r}, \\
\mathcal{H}_{n}=\left[\begin{array}{cc}
\varepsilon_{n}(-i \boldsymbol{\nabla})-\mu & \Delta_{n} e^{i \theta_{n}}+J_{m} e^{i \theta_{m}} \\
\Delta_{n} e^{-i \theta_{n}}+J_{m} e^{-i \theta_{m}} & -\varepsilon_{n}(-i \boldsymbol{\nabla})+\mu
\end{array}\right], \\
\mathcal{H}_{3}=\left[\begin{array}{cc}
\varepsilon_{3}(-i \boldsymbol{\nabla})-\mu & \Delta_{3} e^{i \theta_{3}} \\
\Delta_{3} e^{-i \theta_{3}} & -\varepsilon_{3}(-i \boldsymbol{\nabla})+\mu
\end{array}\right] .
\end{gathered}
$$

Here $\varepsilon(-i \boldsymbol{\nabla})$ is the kinetic-energy operator derived from the corresponding energy dispersion functions $\varepsilon_{n}(\mathbf{k})$ and $\mu$ is the chemical potential. Moreover, $\Delta_{n} e^{i \theta_{n}} \equiv V_{n} \psi_{n}$ where $\psi_{n} \equiv \int d \mathbf{r}\left\langle\psi_{n\rfloor}(\mathbf{r}) \psi_{n \uparrow}(\mathbf{r})\right\rangle$ is the order parameter of superconductor $n=1,2,3$ and $V_{n}$ is the corresponding strength of the pairing potential. Similarly, $J_{m} e^{i \theta_{m}} \equiv I \psi_{m}(m \neq n=1,2)$ encodes the pairing field in band $n$ due to electrons pairing in band $m$ (interband Josephson effect) and $I$ is the strength of the coupling between the two bands. ${ }^{10}$ The two-band Hamiltonian densities $\mathcal{H}_{n=1,2}$ are derived from the two-band model Hamiltonian of Ref. 10. In this Hamiltonian [Eq. (1) therein], there is only intraband pairing (and no interband pairing) and the electrons in the two bands are coupled only through an effective Josephson coupling.

The tunneling part $H_{I}$ contains hopping between the impurity to each one of the three superconductors (with different strengths $t_{n=1,2,3}$ ), which occurs at a single point. Finally, the strong correlation part has the usual structure of an Anderson impurity Hamiltonian. Using again Nambu spinor notation $C=\left[c_{\uparrow}^{\dagger}, c_{\downarrow}\right]$ we have

$$
\begin{gathered}
H_{\mathrm{I}}=-\sum_{n=1}^{3} t_{n}\left[\Psi_{n}^{\dagger}(\mathbf{0}) \tau_{3} C+C^{\dagger} \tau_{3} \Psi_{n}(\mathbf{0})\right], \\
H_{\mathrm{imp}}=\bar{\varepsilon} C^{\dagger} \tau_{3} C+\frac{1}{2} U\left[C^{\dagger} C\right]^{2}, \quad \bar{\varepsilon}=\varepsilon+\frac{U}{2} .
\end{gathered}
$$

The procedure for calculating the free energy $\mathcal{F}(\boldsymbol{\theta})$ is a modified version of the algorithm used in Refs. 15 and 17. It involves an Euclidean path integral for the partition function $Z=e^{-\beta \mathcal{F}}(\mathcal{F}$ is the free energy), in terms of Grassman fields and employing Hubbard-Stratonovich transformation for treating the quartic term in $H_{\mathrm{imp}}$ at the expense of an additional integration on a new field $\gamma$. The latter is carried out within the saddle-point approximation leading to a selfconsistent equation (Hartree-Fock approximation).

Beside the density of states at the Fermi energy $N(\mu)$ (assumed constant) and the impurity level (partial) widths $\Gamma_{n}=\pi t_{n}^{2} N(\mu)$ for the superconductor $n=1,2,3$, the basic input quantities are defined below [with $\omega=\omega_{k}$ $=(2 k+1) \pi T(k=0,1,2, \ldots)$ a Matsubara frequency at temperature $T=1 / \beta$ and $\left.\Sigma_{\omega} f(\omega) \equiv \Sigma_{k} f\left(\omega_{k}\right)\right]$

$$
\begin{gathered}
\alpha_{n=1,2}(\omega)=\Gamma_{n}\left[\Delta_{n}^{2}+J_{m}^{2}+2 \Delta_{n} J_{m} \cos \left(\theta_{1}-\theta_{2}\right)+\omega^{2}\right]^{-1 / 2}, \\
\alpha_{3}=\Gamma_{3}\left[\Delta_{3}^{2}+\omega^{2}\right]^{-1 / 2}, \quad \eta(\omega)=\omega\left[1+\sum_{n=1}^{3} \alpha_{n}(\omega)\right] . \\
q_{1}(\omega)=\alpha_{1}(\omega) \Delta_{1}+\alpha_{2}(\omega) J_{1}, \\
q_{2}(\omega)=\alpha_{2}(\omega) \Delta_{2}+\alpha_{1}(\omega) J_{2}, \quad q_{3}(\omega)=\alpha_{3}(\omega) \Delta_{3}, \\
F(\omega ; \boldsymbol{\theta}) \equiv \sum_{n=1}^{3} q_{n}^{2}+2 \sum_{n \neq n^{\prime}} q_{n} q_{n^{\prime}} \cos \left(\theta_{n}-\theta_{n}^{\prime}\right) .
\end{gathered}
$$

The self-consistent equation for the field $\gamma$ (analogous to Eq. 7 in Ref. 15 or Eq. 12 in Ref. 17) reads

$$
\frac{1}{2 U}-T \sum_{\omega} \frac{\left[\gamma^{2}+\eta(\omega)^{2}-\bar{\varepsilon}^{2}-F(\omega, \boldsymbol{\theta})\right]}{\left[\gamma^{2}-\eta(\omega)^{2}-\bar{\varepsilon}^{2}-F(\omega, \boldsymbol{\theta})\right]^{2}+4 \gamma^{2} \eta(\omega)^{2}}=0,
$$

whose solution $\bar{\gamma}(\boldsymbol{\theta})$ is used below.

\section{Choice of parameters}

The choice of fixed parameters

$$
\Delta_{1,3}=1.0, \quad \Delta_{2}=0.8, \quad \Gamma_{1}=0.5, \quad \Gamma_{2}=\Gamma_{3}=0.2, \quad \varepsilon=-2
$$

is guided by the need to break the $1 \leftrightarrow 2$ symmetry since it was shown ${ }^{13}$ that for noticeable different Josephson tunneling strengths $\Gamma_{1} \neq \Gamma_{2}$ and for $U=0$, there is no TRVGS in a $2 B-1 B$ junction while here we intend to show that for $U \neq 0$ it does exist. Since it is rather difficult to tailor the ratio such that $\Gamma_{1} / \Gamma_{2} \gg 1$ we adopt a reasonable ratio of 2.5 which proves to be sufficient for our purpose. Otherwise, the 

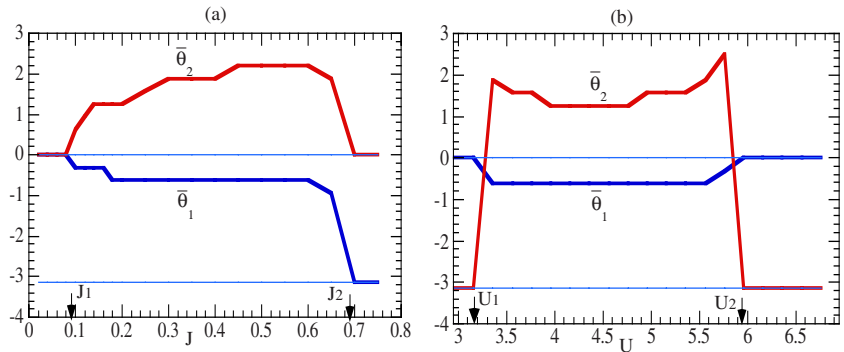

FIG. 2. (Color online) Phases $\bar{\theta}_{1,2}$ of the ground state: (a) as function of $J$ for $U=4$ and (b): as function of $U$ for $J=0.2$. Other parameters are given in Eq. (8). The ground states for $J<J_{1}$ and $J>J_{2}$ (marked by arrows) are $(0,0)$ and $(0, \pi)$ (both are TRCGS) while for $J_{1}<J<J_{2}$ there is a phase of TRVGS. The ground states for $U<U_{1}$ and $U>U_{2}$ (marked by arrows) are $(0, \pi)$ (both are TRCGS) while for $U_{1}<U<U_{2}$ there is a phase of TRVGS.

values of the energy parameters (expressed in terms of $\Delta_{1}$ $=1$ ) are reasonable. Finally, we found it more convenient to work at finite temperature $T=0.0005$ (at this range the sensitivity to temperature is negligible).

\section{ANALYSIS OF THE RESULTS}

The free energy associated with the impurity is the coefficient of $-\beta$ in the exponent of the partition function $Z=e^{-\beta \mathcal{F}}$ and the formalism described above yields for it the following expression:

$$
\begin{gathered}
\mathcal{F}(\boldsymbol{\theta})=\frac{\bar{\gamma}^{2}}{2 U}+\bar{\varepsilon}-\frac{T}{2}, \\
\sum_{\omega} \ln \left\{\beta^{4}\left[\bar{\gamma}^{2}-\eta(\omega)^{2}-\bar{\varepsilon}^{2}-F(\omega, \boldsymbol{\theta})\right]^{2}+4 \bar{\gamma}^{2} \eta(\omega)^{2}\right\} .
\end{gathered}
$$

Employing gauge invariance and setting $\theta_{3}=0$, the minimum $\overline{\boldsymbol{\theta}}=\left(\bar{\theta}_{1}, \bar{\theta}_{2}, 0\right)$ of $\mathcal{F}(\boldsymbol{\theta})$ is located. If $\sin \bar{\theta}_{1}=\sin \bar{\theta}_{2}=0$ one evidently has a TRCGS. On the other hand, when the above condition is not satisfied, one has a TRVGS. In the absence of Anderson impurity and close to perfect symmetry $\delta \approx 1$ it occurs for $J>0$ at $\bar{\theta}_{1} \approx-\bar{\theta}_{2} \approx \frac{\pi}{2}$. For $U \neq 0$ the property $\bar{\theta}_{1} \approx-\bar{\theta}_{2} \approx \frac{\pi}{2}$ is expected to be somewhat modified and regions with $\sin \bar{\theta}_{1}, \sin \bar{\theta}_{2} \neq 0$ are those where TRVGS is realized. In Fig. 2(a) the phases $\bar{\theta}_{1,2}$ are displayed as function of $J$ for fixed $U$ while in Fig. 2(b) they are displayed as function of $U$ for fixed $J$. In each case we can identify three regions, left, right, and middle. For the first two we have $\sin \bar{\theta}_{1}=\sin \bar{\theta}_{2}=0$ corresponding to a TRCGS. In between we identify a phase with $\sin \bar{\theta}_{1} \sin \bar{\theta}_{2}<0$ which clearly indicates a TRVGS.

Perhaps an appropriate candidate for identifying such an extraordinary ground state is by inspecting the behavior of the impurity magnetization as function of $J$ and $U$ in the TRVGS phase and compare it with its characteristics in the TRCGS. This is displayed in Fig. 3 which, beside the impurity magnetization depicts also the ground-state energy. The conclusion from Fig. 3 is that while the ground-state energy

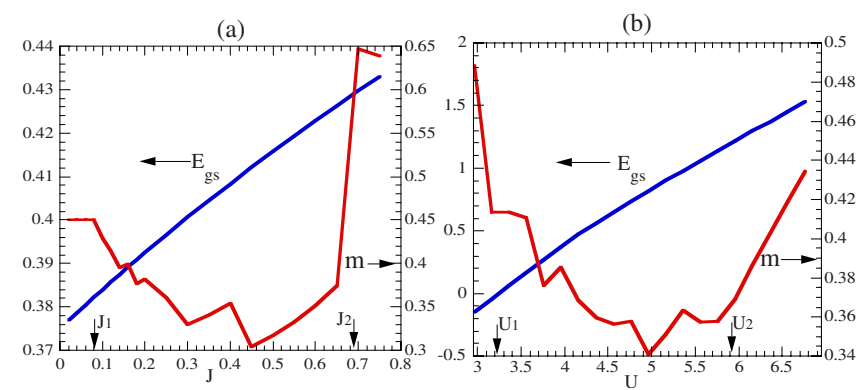

FIG. 3. (Color online) Left scale (right scale): ground-state energy (impurity magnetization) (a) as function of $J$ for $U=4$ and (b) as function of $U$ for $J=0.2$. Other parameters are given in Eq. (8). While the energy is a very smooth function the magnetization has a deep minimum in the TRVGS phase and its jumps correspond to the configuration $\left(\bar{\theta}_{1}, \bar{\theta}_{2}\right)$ of the ground state displayed in Fig. 2.

is smooth across the TRCGS-TRVGS phase boundary the impurity magnetic moment undergoes a sizable reduction in the TRVGS phase compared with its value in the TRCGS phase.

The occurrence of TRVGS implies the existence of nonzero Josephson currents $\mathcal{J}_{n 3}(n=1,2)$ between the coupled superconductors $(1,2)$ and the third superconductor 3 even in the absence of magnetic field. The net Josephson current $\mathcal{J}=\mathcal{J}_{13}+\mathcal{J}_{23}$ should vanish though. Recall that in a junction with single-band superconductors $\mathcal{J}=\frac{e}{\hbar} \frac{\partial E_{g s}(\cos \eta)}{\partial \eta}$ where $\eta$ is the order-parameter phase difference between left and right superconductors. Vanishing of the current (in the absence of an external magnetic field) is associated with the fact that the ground state always occurs at $\eta=0$ (zero junction) or $\eta=\pi$ ( $\pi$ junction). For the $2 B-U-1 B$ studied here the situation is distinct: there are nonzero currents across the junction but their sum vanishes. The derivation of the total current $\mathcal{J}$ from $\mathcal{F}(\boldsymbol{\theta})$ reads

$$
\begin{aligned}
\mathcal{J}= & \frac{e}{\hbar} \frac{\partial \mathcal{F}(\boldsymbol{\theta})}{\partial \theta_{3}}=-\frac{e}{\hbar}\left[\frac{\partial \mathcal{F}(\boldsymbol{\theta})}{\partial\left(\theta_{1}-\theta_{3}\right)}+\frac{\partial \mathcal{F}(\boldsymbol{\theta})}{\partial\left(\theta_{2}-\theta_{3}\right)}\right] \equiv \mathcal{J}_{13} \\
& +\mathcal{J}_{23}, \quad(\text { evaluated at } \boldsymbol{\theta}=\overline{\boldsymbol{\theta}}) .
\end{aligned}
$$

The currents $\mathcal{J}_{13}$ and $\mathcal{J}_{23}$ are displayed in Fig. 4, and indeed, $\mathcal{J}_{13} \sim-\mathcal{J}_{23}$ are both nonzero in the TRVGS. There is a very small deviation of the net Josephson current from zero. This
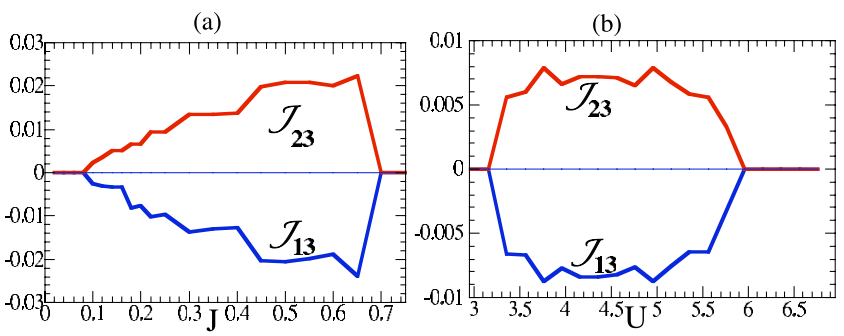

FIG. 4. (Color online) Josephson currents $\mathcal{J}_{13}$ and $\mathcal{J}_{23}$ (in units $\frac{e \hbar}{\Delta}$ ) (a) as function of $J$ for $U=4$ and (b) as function of $U$ for $J=0.2$. Other constants are as in Eq. (8). The jumps correspond to the configuration $\left(\bar{\theta}_{1}, \bar{\theta}_{2}\right)$ of the ground state displayed in Fig. 2. 
is probably an artifact of the assumption that $\Delta_{1,2,3}$ is constant in space (as was noticed in Ref. 13).

\section{DISCUSSION}

To understand the mechanism which drives the formation of the TRVGS state, we recall the case of single-band superconductor Josephson junction. ${ }^{15}$ It was found that the effective Josephson coupling between the two superconductors mediated through the Anderson Impurity changes sign when $U$ goes through a critical value $U_{c}$. The two superconductors are in phase $(\eta=0)$ at $U<U_{c}$ and are out of phase $(\eta=\pi)$ at $U>U_{c}$. The transition can be described phenomenologically by a Josephson free energy $F_{\mathrm{Jos}}\left(\theta_{1}-\theta_{2}\right) \sim T(U) \cos \left(\theta_{1}-\theta_{2}\right)$ where $T(U)$ changes sign at $U=U_{c}$. We now replace one of the superconductors by a two-band superconductor. In this case the effective Josephson coupling $F_{\text {Jos }}$ between the different $\theta_{n}$ 's generated by the impurity has the form

$$
\begin{aligned}
F_{\mathrm{Jos}} \sim & T_{13}(U, J) \cos \left(\theta_{1}-\theta_{3}\right)+T_{23}(U, J) \cos \left(\theta_{2}-\theta_{3}\right) \\
& +T_{12}(U, J) \cos \left(\theta_{1}-\theta_{2}\right)+\sum_{n, m} W_{m n}(U, J) \\
& \times\left(\cos \left(\theta_{n}\right) \cos \left(\theta_{m}\right)\right)+\text { higher order terms, }
\end{aligned}
$$

where $T_{13}(U)$ and $T_{23}(U)$ become small and change sign around $U \sim U_{c}$ and higher-order terms in $F_{\text {Jos }}$ becomes important in determining the phase structure if $T_{12} \sim J$ is also small. The higher-order Josephson terms arises from $\alpha_{n}(\omega)$ 's and $F(\omega ; \boldsymbol{\theta})$ in Eq. (6) which has much more complicated structure than the case of one-band superconductor ${ }^{15}$ and have comparable magnitudes. Apparently, the more complicated phase structure allowed in the $2 B-U-1 B$ Josephson junction leads to appearance of more local extrema of free energy $F_{\mathrm{Jos}}$ in the $\left(\theta_{1}, \theta_{2}\right)$ plane at values other than $(0, \pi)$, which is in sharp contrast to the $1 B-U-1 B$ system where only one phase angle $\theta=\theta_{1}-\theta_{2}$ appears $^{15}$ and local extrema exist only at $\theta=0, \pi$. The appearance of these new energy extrema for a large range of parameters $W_{m n}$ can be demonstrated by minimizing the effective free energy, Eq. (11), (without the higher-order terms) around the critical region $U \sim U_{c}$ and $J \sim J_{c}$. As $U$ increases these local extrema in $F_{\text {Jos }}$ becomes global minima in free energy leading to the appearance of TRVGS around the critical region as shown in Figs. 2(a) and 2(b). We note from Figs. 2(a), 2(b), 3(a), and 3(b) that as $J$ and $U$ change there exists a series of mean-field solutions with different values of $\theta_{1}$ and $\theta_{2}$ and with correspondingly slightly different values of magnetization $m$. The different plausible fractionalized values of magnetization $m$ for different junctions where $J$ and $U$ differ unavoidably is a signal of TRVGS which can be detected experimentally besides the experiments proposed in Ref. 13.

\section{CONCLUSIONS}

The study of $2 B-U-1 B$ carried out here is motivated by the renewed interest in multiband superconductors (stemming from the analysis of the iron-based superconductors). The pertinent physics is fundamentally distinct from that of a $1 B-U-1 B$ system $^{15,17}$ and the $2 B-1 B$ junction discussed in Ref. 13 since the roles of strong correlations $U$ and the coupling $J$ between the two order parameters in the two-band superconductor interlace. It has been shown that there is a finite domain in the $U-J$ plan where a TRVGS emerges even when the coupling ratio $\Gamma_{1} / \Gamma_{2}$ differs significantly from unity. The TRVGS is characterized by small value of the impurity magnetic moment and by unusual values of orderparameter phase differences between superconductors $\left|\bar{\theta}_{i}-\bar{\theta}_{j}\right| \neq 0, \pi$. In the nomenclature of Josephson junctions pertaining to single-band superconductors TRVGS exhibits neither zero nor $\pi$ junction even without a magnetic field. The role of strong correlations in controlling the TR symmetry of the ground state is evidently remarkable. As far as an experimental detection is concerned, beside the experiments proposed in Ref. 13, our preliminary results indicate that some quantities (such as impurity magnetization) undergo a dramatic change as $J$ or $U$ cross the phase boundary, hence a Josephson junction with Anderson impurity can serve as a potential tool for probing the relative phase of the two order parameters in a two-band superconductor (which is a very elusive quantity).

\section{ACKNOWLEDGMENTS}

We would like to thank A. Golub, O. Entin-Wohlman, and F. C. Zhang for fruitful discussions. The work of Y.A. is partially supported by an ISF grant.

\footnotetext{
*yshai@bgu.ac.il

†phtai@ust.hk

${ }^{1}$ J. Nagamatsu, N. Nakagawa, T. Muranaka, Y. Zenitani, and J. Akimitsu, Nature (London) 410, 63 (2001).

${ }^{2}$ A. Y. Liu, I. I. Mazin, and J. Kortus, Phys. Rev. Lett. 87, 087005 (2001).

${ }^{3}$ H. J. Choi, D. Roundy, H. Sun, M. L. Cohen, and S. G. Louie, Phys. Rev. B 66, 020513(R) (2002).

${ }^{4}$ S. Raghu, X.-L. Qi, C.-X. Liu, D. J. Scalapino, and S. C. Zhang, Phys. Rev. B 77, 220503(R) (2008).

${ }^{5}$ P. Lee and X. Wen, Phys. Rev. B 78, 144517 (2008).

${ }^{6}$ X. Feng and T. K. Ng, Phys. Rev B 79, 184503 (2009).

${ }^{7}$ H. Suhl, B. T. Matthias, and L. R. Walker, Phys. Rev. Lett. 3,
}

552 (1959).

${ }^{8}$ V. A. Moskalenko, Fiz. Magn. Mater. 8, 503 (1959).

${ }^{9}$ W. S. Chow, Phys. Rev. 176, 525 (1968).

${ }^{10}$ J. Kondo, Prog. Theor. Phys. 29, 1 (1963).

${ }^{11}$ G. E. Devlin and E. Corenzwit, Phys. Rev. 120, 1964 (1960).

${ }^{12}$ O. Entin-Wohlman and Y. Imry, Phys. Rev. B 40, 6731 (1989).

${ }^{13}$ T. K. Ng and N. Nagaossa, arXiv:cond-mat/08 (unpublished).

${ }^{14}$ S. Mukhopadhyay et al. arXiv:0903.0674 (unpublished).

${ }^{15}$ A. V. Rozhkov and D. P. Arovas, Phys. Rev. Lett. 82, 2788 (1999).

${ }^{16}$ A. A. Clerk and V. Ambegaokar, Phys. Rev. B 61, 9109 (2000).

${ }^{17}$ Y. Avishai and A. Golub, Phys. Rev. B 61, 11293 (2000).

${ }^{18}$ B. I. Spivak and S. A. Kivelson, Phys. Rev. B 43, 3740 (1991). 